\title{
On the Continuity Condition for the Higher-Order VSIE-MoM Formulation
}

\author{
Kim, Oleksiy S.; Jørgensen, Erik; Meincke, Peter; Breinbjerg, Olav
}

Published in:

IEEE Antennas and Propagation Society International Symposium, Monterey, California, June 20-25

Link to article, DOI:

10.1109/APS.2004.1330239

Publication date:

2004

Document Version

Publisher's PDF, also known as Version of record

Link back to DTU Orbit

Citation (APA):

Kim, O. S., Jørgensen, E., Meincke, P., \& Breinbjerg, O. (2004). On the Continuity Condition for the HigherOrder VSIE-MoM Formulation. In IEEE Antennas and Propagation Society International Symposium, Monterey, California, June 20-25 (pp. 4048-4051). IEEE. https://doi.org/10.1109/APS.2004.1330239

\section{General rights}

Copyright and moral rights for the publications made accessible in the public portal are retained by the authors and/or other copyright owners and it is a condition of accessing publications that users recognise and abide by the legal requirements associated with these rights.

- Users may download and print one copy of any publication from the public portal for the purpose of private study or research.

- You may not further distribute the material or use it for any profit-making activity or commercial gain

- You may freely distribute the URL identifying the publication in the public portal 


\section{On the Continuity Condition for the Higher-Order VSIE-MoM Formulation}

Oleksiy S. Kim¹ ${ }^{* 1}$ Erik Jørgensen ${ }^{2}$, Peter Meincke ${ }^{1}$, and Olav Breinbjerg ${ }^{1}$

${ }^{1} \emptyset$ rsted-DTU, Section for Electromagnetic Systems, Building 348

Technical University of Denmark, DK-2800 Kgs. Lyngby, Denmark, $\{$ osk,pme,ob\}@oersted.dtu.dk

${ }^{2}$ TICRA, Læderstræde 34, DK-1201 Copenhagen K, Denmark, ej@ticra.com

\section{Introduction}

The volume-surface integral equation (VSIE) technique involves a system of two coupled equations for a dielectric volume and a perfectly electrically conducting (PEC) surface. At those parts where the PEC surface is in contact with the dielectric material, an additional equation enforcing the continuity between the surface current density on the PEC surface and the normal component of the electric flux density in the dielectric can be introduced. The explicit enforcement of the continuity condition not only allows the number of unknowns in the method of moment (MoM) solution of the VSIE to be reduced, but also ensures a good accuracy of the solution. In the case of low-order basis functions (RWG or rooftop) the latter property is not very pronounced. Due to the local character of the basis functions, an implicit (numerical) enforcement of the continuity condition is quite accurate [1], even for the problems involving metallic edges where the surface current density has a singular behavior. Thus, in accelerated solvers, such as the fast multipole method (FMM/MLFMM), the continuity condition is usually ignored for the sake of implementation simplicity [2].

The situation differs for higher-order basis functions that can be defined on a large domain (up to 1-2 $\lambda$ ). Any inaccuracy in the integration of the MoM procedure affects a large number of basis functions defined on the same domain. Thus, if the continuity condition is satisfied numerically, high integration precision is required to make the normal component of the electric flux density in the dielectric conform to the local behavior of the surface current density at the edge of the adjoining PEC surface. However, the accuracy of the solution can be maintained without increasing the integration order if the continuity condition is imposed explicitly. In the present paper, we show by numerical examples advantages of the explicit enforcement of the continuity condition at the interface between metal and dielectric when higher-order hierarchical basis functions are utilized.

\section{Higher-Order Method of Moments for the VSIE}

The volume-surface integral equation formulation for the electric field is based on two coupled equations. The first equation expresses the total electric field $\mathbf{E}(\mathbf{r})$ as a sum of the incident electric field $\mathbf{E}^{i}(\mathbf{r})$ and the scattered electric feld $\mathbf{E}^{s}(\mathbf{r})$ in the dielectric volume $\mathrm{V}$

$$
\mathbf{E}(\mathbf{r})=\mathbf{E}^{i}(\mathbf{r})+\mathbf{E}_{V}^{s}(\mathbf{r})+\mathbf{E}_{S}^{s}(\mathbf{r}), \quad \mathbf{r} \in V,
$$

-This work was supported by the Danish Technical Research Council and the Danish Center for Scientific Computing. 
where $\mathbf{E}_{V}^{s}(\mathbf{r})$ and $\mathbf{E}_{S}^{s}(\mathbf{r})$ are the electric field scattered by the unknown induced electric volume current density $\mathbf{J}_{V}(\mathbf{r})$ and the electric surface current density $\mathbf{J}_{S}(\mathbf{r})$, respectively. In our formulation the electric flux density $\mathbf{D}(\mathbf{r})$ is used as the unknown instead of $\mathbf{J}_{V}\langle\mathbf{r})$ because the normal component of $\mathbf{D}(\mathbf{r})$ is continuous across the boundary between two different dielectric materials. The second equation in the VSIE imposes the boundary condition for the electric field on the PEC surface $S$

$$
\mathbf{E}^{i}(\mathbf{r})=-\mathbf{E}_{V}^{s}(\mathbf{r})-\mathbf{E}_{S}^{s}(\mathbf{r}), \quad \mathbf{r} \in S .
$$

In this paper we employ higher-order hierarchical Legendre vector basis functions [3] for the discretization of the VSIE. Previously, these functions have been successfully applied to the analysis of metallic objects in free space [3] and in layered media [4] by surface integral equations, and to the analysis of dielectric objects by volume integral equations [5]. The discretization of the geometry of the object is carried out by curvilinear hexahedral and quadrilateral elements. In each element the unknown function, $\mathbf{D}(\mathbf{r})$ or $\mathbf{J}_{S}(\mathbf{r})$, is expanded in the curvilinear coordinate system $(u, v, w)$ in terms of the higher-order hierarchical Legendre basis functions $[3,5]$ as

$$
D^{\xi}=\frac{1}{A} \sum_{m=0}^{M^{\xi}} \sum_{n=0}^{N^{\zeta}} \sum_{q=0}^{Q^{\eta}} a_{m n q}^{\xi} \tilde{P}_{m}(\xi) P_{n}(\zeta) P_{q}(\eta)
$$

where $(\xi, \zeta, \eta)$ is $(u, v, w),(v, w, u)$ or $(w, u, v)$, and

$$
J_{S}^{\xi}=\frac{1}{A} \sum_{m=0}^{M^{\xi}} \sum_{n=0}^{N^{\zeta}} b_{m n}^{\xi} \tilde{P}_{m}(\xi) P_{n}(\zeta)
$$

where $(\xi, \zeta)$ is $(u, v)$ or $(v, u)$ with $-1 \leq u, v, w \leq 1$. Herein

$$
\tilde{P}_{m}(\xi)= \begin{cases}1-\xi, & m=0 \\ 1+\xi, & m=1 \\ P_{m}(\xi)-P_{m-2}(\xi), & m \geq 2,\end{cases}
$$

$A$ is the Jacobian of the parametric transformation, $P_{m}(\xi)$ are Legendre polynomials, $a_{m n q}$ and $b_{m n}$ are unknown coefficients, and $M^{\xi}, N^{\zeta}$, and $Q^{\eta}$ denote the expansion orders along the parametric directions. Galerkin's testing procedure is applied to transform the integral equation into a system of linear equations.

\section{Imposing the Continuity Condition}

At a metal-dielectric boundary, the surface charge density $\rho_{S}(\mathbf{r})$ and the electric flux density must satisfy the continuity condition

$$
\hat{\mathbf{n}} \cdot \mathbf{D}(\mathbf{r})=\rho_{S}(\mathbf{r})=\frac{j}{\omega} \nabla \cdot \mathbf{J}_{S}(\mathbf{r})
$$

where $\hat{\mathbf{n}}$ is a unit vector normal to the boundary. Making use of the favorable orthogonality properties of the higher-order hierarchical Legendre basis functions [3], the surface charge density $\rho_{S}$ as well as $\hat{\mathbf{n}} \cdot \mathbf{D}$ can be expressed in terms of orthogonal functions [5]. Consequently, the unknown coefficients for the component of the electric flux density normal to the PEC surface (e.g., the component $D^{w}$ ) are explicitly expressed in terms of the coefficients for the current $\mathbf{J}_{S}$ on the PEC surface as

$$
a_{1 n q}^{w}=\frac{j}{2 \omega} \begin{cases}b_{10}^{u}-b_{00}^{u}+b_{10}^{u}-b_{00}^{v}, & n=q=0 . \\ (2 n+1) b_{(n+1) 0}^{u}+b_{1 n}^{v}-b_{0 n}^{v}, & n>0 ; q=0 \\ b_{1 q}^{u}-b_{0 q}^{u}+(2 q+1) b_{(q+1) 0}^{v}, & n=0 ; q>0 \\ (2 n+1) b_{(n+1) q}^{u}+(2 q+1) b_{(q+1) n}^{v}, & n>0 ; q>0 .\end{cases}
$$




\section{Numerical Examples}

First, scattering by a PEC sphere of radius $R=1.5 \lambda_{0}$ coated with a dielectric material of thickness $0.1 \lambda_{0}$ and $\epsilon_{\tau}=1.5-j 0.5$ is considered. This object is investigated because it does not contain edges. The sphere is discretized by 54 curvilinear quadrilaterals for the PEC surface and 54 curvilinear hexahedra for the dielectric coating. A maximum of $M^{\xi}=4$ is used when expanding the unknown functions $\mathrm{J}_{S}(\mathrm{r})$ and $\mathbf{D}(\mathbf{r})$. The radar cross section (RCS) computed with and without enforcement of the continuity condition (6) is presented in Fig. 1. In both cases the orders of the Gauss quadrature rules employed in the integration are the same. The exact Mie series solution is used as a reference. It is observed that the enforcement of the continuity condition does not influence the accuracy of the solution in this case. Nevertheless, 864 unknowns out of 5184 can be removed when (6) is used.

Second, a frequency selective surface (FSS) formed by a 5 by 5 array of dipoles printed on a finite dielectric substrate is analyzed. This configuration contains edges. The substrate has $\epsilon_{r}=3$ and thickness $0.037 \mathrm{~mm}$. A unit element of the FSS is shown in Fig. 2. The FSS is illuminated by a $y$-polarized plane wave propagating from the direction given by $\theta^{i}=30^{\circ}$ and $\phi^{i}=0^{\circ}$. The RCS is calculated in the $x z$-plane at $\theta^{i}=150^{\circ}$ and $\theta^{i}=180^{\circ}$ with maximum expansion order $M^{\xi}=3$ for $\mathbf{D}(\mathbf{r})$ and $M^{\xi}=2$ for $\mathbf{J}_{S}(\mathbf{r})$. Fig. 3 shows the results of our higher-order technique with and without the continuity condition (6) enforced. The results obtained by using a fine discretization and first-order basis functions (rooftop) are also shown as a reference. The low-order solution is not influenced much by the enforcement of the continuity condition. However, as opposed to the previous example, the explicitly imposed continuity condition now significantly improves the higher-order solution. Both results with higher-order basis functions in Fig. 3 are obtained with the same precision of the Gauss quadrature integration. If the continuity condition is not enforced in the higher-order technique, the solution can be improved by increasing the integration accuracy. Fig. 4 shows the convergence of the result as the orders of the Gauss quadrature rules used in the solution are increased in steps by one from 0 to 3 . It is noted that the MoM matrix filling time considerably increases with the increment of the integration order.

\section{Conclusion}

A higher-order MoM solution of the VSIE based on the higher-order hierarchical Legendre basis functions has been presented. The technique is suitable for solving electromagnetic problems involving composite metallic-dielectric objects. Numerical examples for a finite FSS show that the explicit enforcement of the continuity condition at a metallic-dielectric interface significantly improves the solution if higher-order basis functions are defined on rather large domains with metallic edges present. This improvement manifests itself in reducing the number of unknowns and reducing the MoM matrix filling time for the same accuracy achieved in comparison with the solution in which the continuity condition is ignored.

\section{References}

[1] C. Yu and C.-C. Lu, "Analysis of curved frequency selective surfaces using the hybrid volume-surface integral equation approach," in Proceedings of the 2003 Int. Symp. on Antennas and Propagation, vol. 2, Columbus, Ohio, June 2003, pp. 809-812. 
[2] C.-C. Lu, "Volume-surface integral equation," in Fast and efficient algorithms in computational electromagnetics, E. M. W.C. Chew, J.-M. Jin and J.Song, Eds. Boston-London: Artech House, 2001, pp. 487-540.

[3] E. Jørgensen, J. L. Volakis, P. Meincke, and O. Breinbjerg, "Higher-order hierarchical Legendre basis functions for electromagnetic modeling," 2003, accepted June 2003 to IEEE Trans. on Antennas and Propagation.

[4] E. Jørgensen, O. S. Kim, P. Meincke, and O. Breinbjerg, "Higher-order hierarchical discretization scheme for surface integral equations for layered media," 2003, accepted July 2003 to IEEE Trans. Geosci. Remote Sensing.

[5] O. S. Kim, E. Jørgensen, P. Meincke, and O. Breinbjerg, "Analysis of scattering by inhomogeneous dielectric objects using higher-order hierarchical MoM," Sevastopol, Ukraine, pp. 113-116, September 2003.
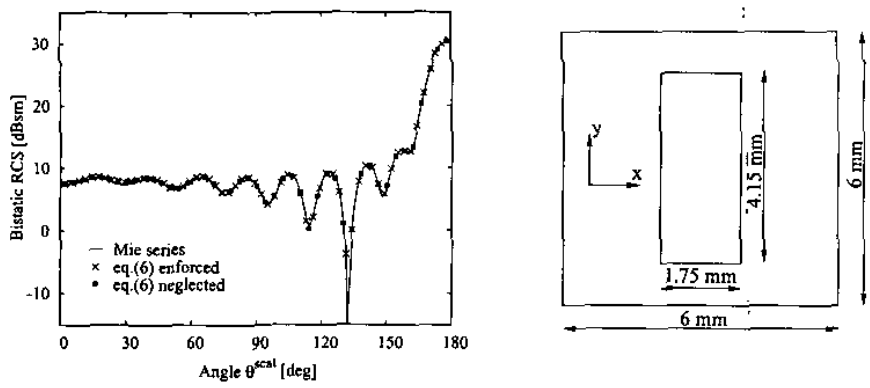

Figure 1: Bistatic RCS (E-plane) for a Figure 2: The FSS unit element. coated PEC sphere.
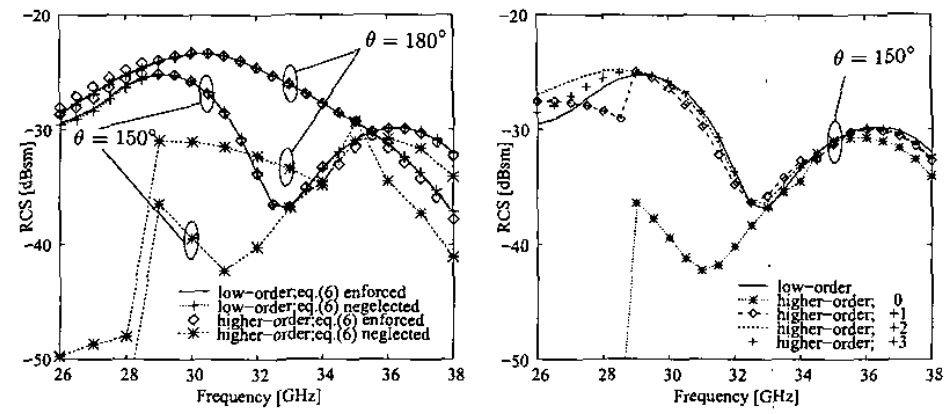

Figure 3: The RCS for a 5 by 5-element Figure 4: Convergence of the solution for FSS.

the FSS as the integration precision increases. The continuity condition is not enforced. 